\title{
Magnetic Properties of FeSeTe Compound Crystallized from Liquid Phase
}

\author{
S.J. LeWANdowski ${ }^{a}$, M. Berkowski ${ }^{a}$, V.Yu. TAREnKOV ${ }^{b}$, A.I. D'YACHEnKo ${ }^{b}$, \\ A. Abal'oshev ${ }^{a}$, I. Abal'osheva ${ }^{a}$, D. Gawryluk $^{a}$, V. Domukhovski $^{a}$, \\ S.L. SIDOROV ${ }^{b}$ AND D.I. BOICHENKO ${ }^{b}$ \\ ${ }^{a}$ Instytut Fizyki PAN, al. Lotników 32/46, 02-668 Warszawa, Poland \\ ${ }^{b}$ Donetsk Institute for Physics and Engineering, R. Luxemburg 72, 83114 Donetsk, Ukraine
}

We report on measurements of samples with nominal composition $\mathrm{FeSe}_{0.5} \mathrm{Te}_{0.5}$, crystallized by the Bridgman method. Magnetic and transport properties of the samples were examined. The measurements confirm the coexistence of ferromagnetism and superconductivity below the superconducting transition temperature. The ferromagnetic contribution to magnetization, estimated at $10 \%$, might be caused by the presence of ferrimagnetic $\mathrm{Fe}_{7} \mathrm{Se}_{8}$, which occupies about $10 \%$ of sample volume. From the Andreev spectroscopy we found superconducting energy gap $\Delta=2.6 \mathrm{meV}$ at $T=4.2 \mathrm{~K}$, and from magnetization measurements the critical temperature $T_{\mathrm{c}}=15.8 \mathrm{~K}$. The critical current density in magnetic field $H=4 \mathrm{kOe}$, determined from magnetization measurements, is $j_{\mathrm{c}}=(1-2) \times 10^{4} \mathrm{~A} / \mathrm{cm}^{2}$ and weakly depends on magnetic field intensity.

PACS numbers: 74.25.Ha, 74.45.+c, 74.62.Bf, 74.70.Xa, 75.30.Cr

\section{Introduction}

The unexpected discovery of superconductivity in the layered iron-based quaternary oxypnictide $\mathrm{LaOFeAs}_{1-x} \mathrm{~F}_{x}$ with critical temperature $T_{\mathrm{c}}=26 \mathrm{~K}$ [1] started a race to find new Fe-based superconducting compounds. Very soon, superconductivity was observed in $\beta-\mathrm{FeSe}_{1-x}$ with critical temperature $\approx 8 \mathrm{~K}[2]$. The crystal lattice of $\beta$-FeSe belongs to tetragonal $(P 4 / \mathrm{nmm})$ group of symmetry at room temperature and is composed of $\mathrm{Se}-\mathrm{Fe}_{2}-\mathrm{Se}$ layers [2], i.e. it has the same planar structure as LaOFeAs [3]. Following the FeSe discovery, considerable effort was made to investigate the effect of chemical substitutions, either to the Se-site or the Fe-site, on superconductivity. This led to the discovery of superconductivity in the PbO-type compound $\mathrm{FeSe}_{1-x} \mathrm{Te}_{x}(x=0 \div 1)$ [4]. It was found that superconducting transition temperature increases with Te doping, reaches a maximum of $\approx 15 \mathrm{~K}$ at about $50 \%$ substitution, and then decreases with more Te doping. The low-temperature structural deformation apparently is essential to the coexistence of magnetism and superconductivity in this class of materials. $T_{\mathrm{c}}$ enhancement is well correlated with the structural deformation resulting from Te substitution.

We report here on measurements confirming the coexistence of ferromagnetism and superconductivity in $\mathrm{Fe}\left(\mathrm{Se}_{0.5} \mathrm{Te}_{0.5}\right)$ even below the superconducting transition temperature.

\section{Sample preparation}

The samples were prepared by mixing the appropriate mole amounts of component elements in evacuated

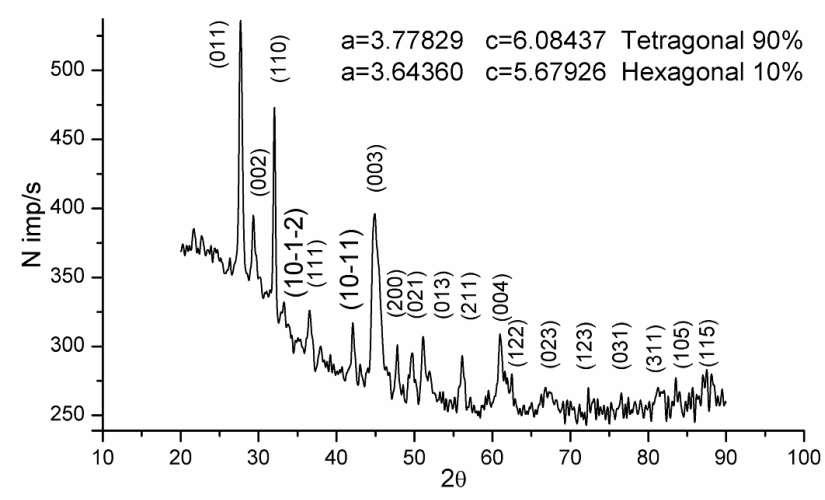

Fig. 1. Diffraction pattern of the sample.

double-walled ampoules, where the synthesis and crystallization by the Bridgman method took place. Here we report on measurements of samples with nominal composition $\mathrm{FeSe}_{x} \mathrm{Te}_{1-x}, x=0.5$, yielding optimal superconducting properties $(x$ reduction increases elementary cell volume and decreases the superconducting transition temperature). Crystallization process was started at $950^{\circ} \mathrm{C}$, and then the ampoules were cooled at the rate of $3^{\circ} \mathrm{C} / \mathrm{h}$ to $750^{\circ} \mathrm{C}$, and from $750^{\circ} \mathrm{C}$ to $350^{\circ} \mathrm{C}$ at $6^{\circ} \mathrm{C} / \mathrm{h}$, and then cooled with the furnace. The resulting ingots had conical part about $10 \mathrm{~mm}$ long evolving into a cylinder of about $8 \mathrm{~mm}$ diameter. Cleaved cone shows elliptical cleavage plane with reflex half-width $\Delta m=360^{\prime \prime}$ at the cone top, changing to $\Delta m=20^{\prime}$ at the base. In the cylindrical part, as the distance from the cone grows, the sample becomes polycrystalline with randomly oriented, well developed crystallites of about $1 \mathrm{~mm}$ size. Lattice 
constants $a$ and $c$ are given in Table, together with volume content of the tetragonal phase, as determined by X-ray methods (see also Fig. 1). The remainder of sample volume is composed of $\mathrm{Fe}_{7} \mathrm{Se}_{8}$ in hexagonal phase.

TABLE

Structural data of the investigated $\mathrm{FeSe}_{0.5} \mathrm{Te}_{0.5}$ sample.

\begin{tabular}{c|c|c|c|c|c}
\hline \hline Composition & Structure & $a[\AA]$ & $c[\AA]$ & $c / a$ & $\begin{array}{c}\% \text { tetrag. } \\
\text { phase }\end{array}$ \\
\hline $\mathrm{FeSe}_{0.5} \mathrm{Te}_{0.5}$ & $P 4 / \mathrm{nmm}$ & 3.7783 & 6.0844 & 1.610 & 90
\end{tabular}

\section{Sample characterization}

We examined magnetic and transport properties of the samples. Initial characterization was carried out by the nondestructive magneto-optical method, which indicated a considerable nonhomogeneity of superconducting properties.

Measurements involving small-area junctions formed by pressing silver $(\mathrm{Ag})$ or indium (In) pellets into $\mathrm{FeSe}_{0.5} \mathrm{Te}_{0.5}$ samples were also carried out. Differentiation of $I-V$ characteristics of the FeSeTe-Ag structure (Fig. 2) shows a well expressed Andreev reflection and yields superconducting gap $\Delta=2.6 \mathrm{meV}$ at $T=4.2 \mathrm{~K}$.

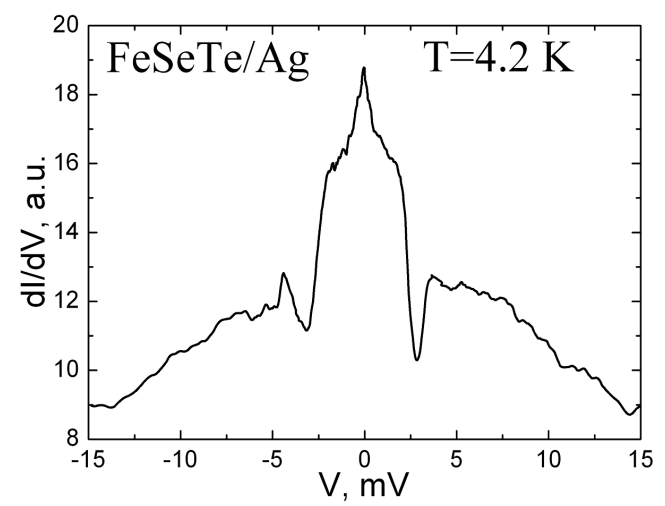

Fig. 2. Conductivity of $\mathrm{FeSeTe}-\mathrm{Ag}$ Andreev junction in liquid helium temperature. The two minima occur at voltages corresponding to the superconducting gap $\Delta=2.6 \mathrm{meV}$, the sharp peak in the neighborhood of $V=0$ may be due to $d$-wave pairing of charge carriers.

\section{Magnetic properties}

Measurements of magnetization $M$ and magnetic susceptibility $\chi$ were carried out in the Quantum Design Physical Property Measurement System (PPMS). The results indicate a coexistence of ferromagnetic and superconducting properties in the investigated material. Above the critical temperature $T_{\mathrm{c}}=15.8 \mathrm{~K}$, the material behaves like a ferromagnet with coercive force $H_{\mathrm{c}} \approx$ 200 Oe (Fig. 3). Below $T_{\mathrm{c}}$, it is a type II superconductor and shows nearly perfect diamagnetism, but still retains some ferromagnetic properties, as evidenced by the lack of symmetry in the $M(H)$ plot in Fig. 4. The critical current density in magnetic field $H=4 \mathrm{kOe}$, determined from $M(H)$ dependence, is $j_{\mathrm{c}}=(1-2) \times 10^{4} \mathrm{~A} / \mathrm{cm}^{2}$ and weakly depends on magnetic field intensity, in agreement with the Kim-Anderson model.

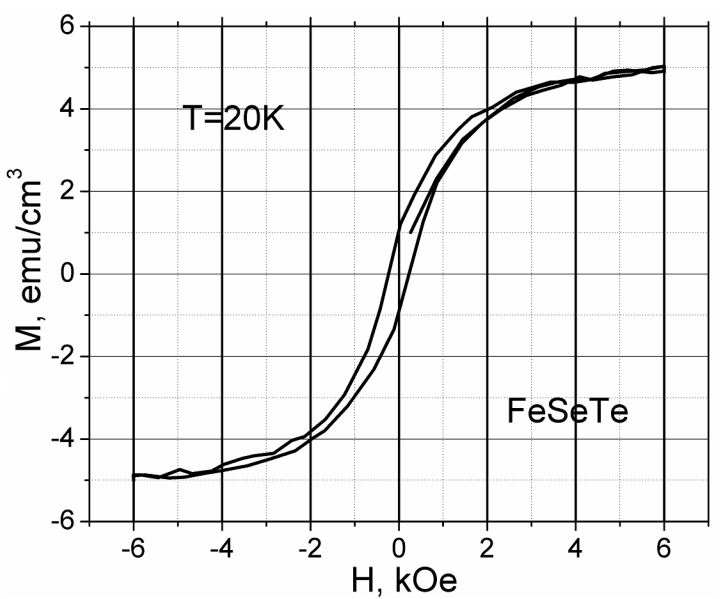

Fig. 3. Hysteresis loop above the critical temperature, clearly showing ferromagnetic properties of the sample.

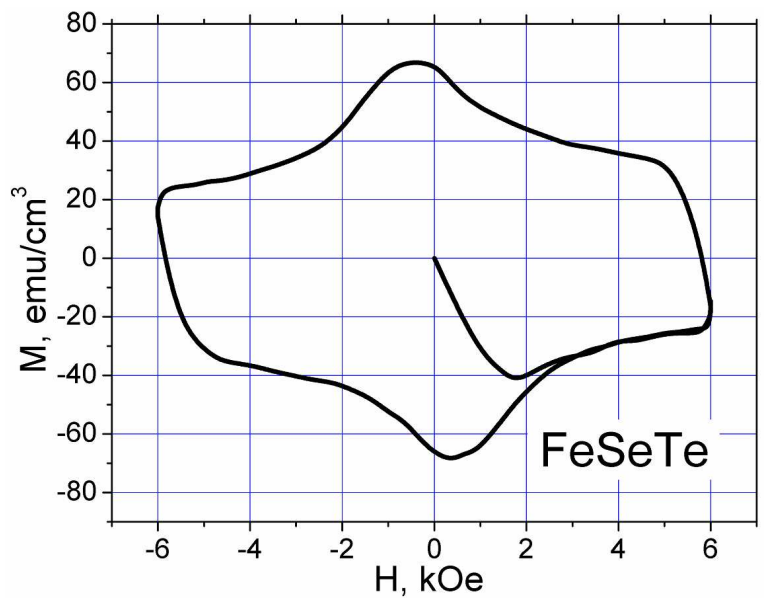

Fig. 4. Hysteresis loop below the superconducting transition temperature. Distortion of the loop with respect to the predictions of critical state model indicates the presence of ferromagnetic regions within the sample volume.

By comparing the experimental $M(H)$ dependence to the critical state model we can estimate that the contribution of ferromagnetic regions amounts to about $10 \%$ of the total sample magnetization. It has been suggested [5] that this contribution is caused by actual composition of the investigated compound being $\mathrm{Fe}_{1+\delta}\left(\mathrm{Se}_{1+x} \mathrm{Te}_{x}\right)$, where $\delta$ denotes excess Fe ions, which occupy partially the interstitial sites, and form planes parallel to the base FeSeTe planes. However, it is more 
probable that it comes from the inclusions of ferrimagnetic [6] $\mathrm{Fe}_{7} \mathrm{Se}_{8}$, which has also planar structure. In any case, we could have superconducting blocks of stoichiometric $\mathrm{FeSe}_{1+x} \mathrm{Te}_{x}$ compound separated by normal regions of ferromagnetic inclusions. As a result, the sample would constitute a Josephson medium for currents flowing along the $c$ axis, while critical state model would remain valid for currents perpendicular to this axis. This would account also for the observed rather broad $R(T)$ and sharp $\chi(T)$ transitions.

The $M(T), R(T)$ and $\chi(T)$ dependences all show well expressed anomalous peaks in the temperature range 120-150 K, similar to those observed in FeSe [5, 7], but in our case the temperature $T_{\mathrm{s}}$ corresponding to this anomaly is about $50 \mathrm{~K}$ higher. Such singularities are usually caused by structural transitions or transitions in magnetic subsystem [5].

\section{Conclusions}

In conclusion, we have successfully synthesized by Bridgman method $\mathrm{FeSe}_{0.5} \mathrm{Te}_{0.5}$ samples of monocrystalline quality and investigated their magnetic and superconducting properties. Magneto-optical observations have shown rather poor homogeneity of superconducting parameters of the samples. From Andreev spectroscopy, the superconducting gap was determined as $\Delta=2.6 \mathrm{meV}$, and some features point to $d$-wave pairing of charge carriers. A probable cause of the observed ferromagnetic properties is the presence of $\mathrm{Fe}_{7} \mathrm{Se}_{8}$ inclusions. We found that magnetization, susceptibility and resistivity peak in the temperature range $120-150 \mathrm{~K}$, indicating a structural or magnetic transition in this range.

\section{Acknowledgments}

This work was partly supported by FunDMS Advanced Grant of European Research Council within the EUFP7 "Ideas" Programme.
The authors express their gratitude to Dr. R. Puźniak for drawing their attention to magnetic properties of $\mathrm{Fe}_{7} \mathrm{Se}_{8}$.

\section{References}

[1] Y. Kamihara, T. Watanabe, M. Hirano, H. Hosono, J. Am. Chem. Soc. 130, 3296 (2008).

[2] F.C. Hsu, J.Y. Luo, K.W. Yeh, T.K. Chen, T.W. Huang, P.M. Wu, Y.C. Lee, Y.L. Huang, Y.Y. Chu, D.C. Yan, M.K. Wu, Proc. Natl. Acad. Sci. USA 105, 14262 (2008).

[3] P. Terzieff, K.L. Komarek, Monatsh. Chem. 109, 651 (1978); W. Schuster, H. Mimer, K.L. Komarek, Monatsh. Chem. 110, 1153 (1979).

[4] K.W. Yeh, T.W. Huang, Y.L. Huang, T.K. Chen, F.C. Hsu, P.M. Wu, Y.C. Lee, Y.Y. Chu, C.L. Chen, J.Y. Luo, D.C. Yan, M.K. Wu, Europhys. Lett. 8437002 (2008); M.K. Wu, F.C. Hsu, K.W. Yeh, T.W. Huang, J.Y. Luo, M.J. Wang, H.H. Chang, T.K. Chen, S.M. Rao, B.H. Mok, C.L. Chen, Y.L. Huang, C.T. Ke, P.M. Wu, A.M. Chang, C.T. Wu, T.P. Perng, Physica C 469, 340 (2009).

[5] W. Bao, Y. Qiu, Q. Huang, M.A. Green, P. Zajdel, M.R. Fitzsimmons, M. Zhernenkov, Minghu Fang, B. Qian, E.K. Vehstedt, Jinhu Yang, H.M. Pham, L. Spinu, Z.Q. Mao, cond.-math 0809.2058 (2008); also in Phys. Rev. Lett. 102, 247001 (2009).

[6] A. Okazaki, J. Phys. Soc. Japan 16, 1162 (1961); E.C. Kim, K.S. Baek, H.N. Ok, J. Korean Phys. Soc. 26, 86 (1993).

[7] L. Li, Z.R. Yang, M. Ge, L. Pi, J.T. Xu, B.S. Wang, Y.P. Sun, Y.H. Zhang, J. Supercond. Nov. Magn. 22, 667 (2009). 